\title{
CHERN-SIMONS-MASLOV CLASSES OF SOME SYMPLECTIC VECTOR BUNDLES
}

\author{
HARUO SUZUKI
}

(Communicated by James E. West)

\begin{abstract}
Let $E_{0}, J_{0}$, and $L_{0}$ be the symplectic $2 n$-vector bundle, the compatible complex operator, and the Lagrangian subbundle that are determined by the $U(n)$-extension of the principal $O(n)$-bundle $U(n) \rightarrow U(n) / O(n)$. We compute the Chern-Simons-Maslov class $\mu^{1}\left(E_{0}, J_{0}, L_{0}\right)$. Then for a trivial symplectic $2 n$-bundle $E$, a compatible complex operator $J$, and a Lagrangian subbundle $L$, we compute Chern-Simons-Maslov classes $\mu^{h}(E, J, L)$ under some condition on the base space of $E$.
\end{abstract}

\section{INTRODUCTION}

A symplectic vector bundle $E$ over a smooth manifold $M$ has a compatible complex structure $J$. If $E$ has a Lagrangian subbundle $L$ then the ChernSimons-Maslov classes $\mu^{h}(E, J, L)$ are defined by Vaisman [V].

If $E$ is a complex extension of a real $n$-vector bundle $L$ then it is canonically a symplectic $2 n$-vector bundle with a Lagrangian subbundle $L$. Suppose $E$ is trivial. Then it is classified by the principal $U(n)$-bundle

$$
\pi_{0}: P_{0}=U(n) \times_{O(n)} U(n) \rightarrow M_{0}=U(n) / O(n) .
$$

Let $E_{0}$ be the symplectic $2 n$-vector bundle corresponding to the $U(n)$ bundle $P_{0} \rightarrow M_{0}, J_{0}$ the natural complex structure in $E_{0}$, and $L_{0}$ the Lagrangian subbundle of $E_{0}$ corresponding to the $O(n)$-bundle $\pi_{0}: U(n) \rightarrow M_{0}$. In this situation, we denote $P_{0}$ by $U_{J_{0}}\left(E_{0}\right)$. In this note, we compute explicitly the class $\mu^{1}\left(E_{0}, J_{0}, L_{0}\right)$ and the classes $\mu^{h}(E, J, L)$ for symplectic vector bundles $E$ classified by $E_{0}$, under some conditions on the base space $M$.

Since $U_{J_{0}}\left(E_{0}\right)$ is a trivial $U(n)$-bundle by the off-diagonal section $s_{0}: M_{0} \rightarrow$ $U_{J_{0}}\left(E_{0}\right)$, the cohomology ring $H^{*}\left(U_{J_{0}}\left(E_{0}\right) ; \mathbf{R}\right)$ is isomorphic to the tensor product $H^{*}\left(M_{0} ; \mathbf{R}\right) \otimes H^{*}(U(n) ; \mathbf{R})$ by the Künneth formula.

Received by the editors June 3,1991; the contents of this paper have been presented at the Annual Meeting of the Mathematical Society of Japan, Yokohama, Japan, April 3, 1991, as a preliminary report.

1991 Mathematics Subject Classification. Primary 57R20; Secondary 58F05, 81S10.

Key words and phrases. Chern-Simons-Maslov classes, symplectic, Lagrangian, connections, curvatures. 
Theorem 1. Let $u \in H^{1}\left(M_{0}\right) \cong \mathbf{Z}$ and $v \in H^{1}(U(n)) \cong \mathbf{Z}$ be canonical generators. Then we have

$$
\mu^{1}\left(E_{0}, J_{0}, L_{0}\right)=\frac{1}{2}(u \otimes 1-2 \otimes v) .
$$

Let $y_{k} \in H^{2 k-1}(U(n) ; \mathbf{R})$ be canonical generators of the cohomology ring $H^{*}(U(n) ; \mathbf{R})$ and $\Delta_{*} y_{2 h-1} \in H^{4 h-3}(U(n) / O(n) ; \mathbf{R})$ denote the canonical generators of the cohomology ring $H^{*}(U(n) / O(n) ; \mathbf{R})$. If $E$ is trivial and it is classified by a map $f: M \rightarrow U(n) / O(n)$, then the pullbacks $\Delta_{E^{*}} y_{2 h-1}=f^{*} \Delta_{*} y_{2 h-1}$ are Maslov classes in the sense of Kamber and Tondeur [KT].

Let $U_{J}(E)$ denote the total space of the principal $U(n)$-bundle associated with $E$. It follows that $H^{*}\left(U_{J}(E) ; \mathbf{R}\right)$ is isomorphic to $H^{*}(M ; \mathbf{R}) \otimes$ $H^{*}(U(n) ; \mathbf{R})$ as $H^{*}\left(U_{J_{0}}\left(E_{0}\right) ; \mathbf{R}\right)$.

Theorem 2. Let $E$ be a symplectic 2 -vector bundle over a manifold $M$, being trivial with respect to a compatible complex structure $J$, and $L \subset E a$ Lagrangian subbundle. If $H^{i}(M ; \mathbf{R})=0$ for $0<i<4 h-3$ then we have

$$
\mu^{h}(E, J, L)=-\Delta_{E *} y_{2 h-1} \otimes 1+1 \otimes y_{2 h-1} \text {. }
$$

In $\S 1$, by following Vaisman [V], we review symplectic $2 n$-vector bundles $E$, compatible complex structures $J$, Lagrangian subbundles $L$, and ChernSimons-Maslov classes $\mu^{h}(E, J, L)$. In $\S 2$, by making use of a flat connection on $U_{J_{0}}\left(E_{0}\right)$ extending the canonical $O(n)$-connection of the bundle $U(n) \rightarrow$ $M_{0}$, representative cocycles of $\mu^{h}\left(E_{0}, J_{0}, L_{0}\right)$ are obtained. In $\S 3$, Theorem 1 is proved. In the last section we show relations between the Chern-SimonsMaslov classes and the Maslov classes in the sense of Kamber and Tondeur and prove Theorem 2.

\section{SyMPLECTIC VECTOR BUNDLES}

Let $\pi_{E}: E \rightarrow M$ be a $C^{\infty}$ symplectic $2 n$-vector bundle over a manifold $M$ of dimension $m$ with its structure defined by a nondegenerate cross-section $\Omega$ of $\wedge^{2} E^{*}$, where $E^{*}$ is the dual bundle of $E$. By taking a complex structure $J$ compatible with $\Omega, E$ becomes a complex $n$-vector bundle. The principal $\mathrm{GL}(n, \mathbf{C})$-bundle associated with $E$ has a $U(n)$-reduction, that is, a principal $U(n)$-bundle $\pi: U_{J}(E) \rightarrow M$.

Let $L \subset E$ be a Lagrangian subbundle, i.e., a real $n$-dimensional subbundle with $\left.\Omega\right|_{L}=0$. Then the structural group of $E$ is reduced to $O(n)$ and one gets a principal $O(n)$-subbundle of $U_{J}(E), \pi: U_{J}(E, L) \rightarrow M$ with equivalent unitary frames $\tilde{e}_{i}=\left(e_{i}-\sqrt{-1} J e_{i}\right) / \sqrt{2}$ for orthonormal basis $e_{i} \in L, i=$ $1, \ldots, n$.

An $O(n)$-connection $\theta$ on $U_{J}(E, L)$ is extended to a $U(n)$-connection on $U_{J}(E)$ naturally. Since the Chern polynomials of odd order vanish on the Lie algebra of $O(n)$, for the curvature form $\boldsymbol{\theta}=d \theta+[\theta, \theta]$ we have $c_{2 h-1}(\boldsymbol{\theta})=0$. $c_{k}(\theta)$ is denoted by $\Delta(\theta) c_{k}$ when the connection $\theta$ is emphasized. It is also denoted by $c_{k}\left(\Theta^{(k)}\right)$ showing the $k$-multilinear property.

Let $\Delta^{1}$ denote the standard 1-simplex. $t \theta$ for $t \in \Delta^{1}$ is a differential 1form on $U_{J}(E) \times \Delta^{1}$, which is not necessarily a connection on the $U(n)$-bundle $U_{J}(E) \times \Delta^{1} \rightarrow M \times \Delta^{1}$. We take a "formal curvature" of $t \theta$,

$$
\overline{\boldsymbol{\Theta}}=d(t \theta)+[t \theta, t \theta]=d t \wedge \theta+\overline{\boldsymbol{\Theta}}_{t}
$$


where $\overline{\boldsymbol{\Theta}}_{t}$ is defined by $\overline{\boldsymbol{\Theta}}_{t}=t d \theta+t^{2}[\theta, \theta]=t \boldsymbol{\Theta}+t(t-1)[\theta, \theta]$.

A suspension form of the Chern form $\Delta(\theta) c_{2 h-1}=c_{2 h-1}(\Theta)$ is defined by

$$
\begin{aligned}
T(\theta) c_{2 h-1} & =-f_{\Delta^{1}} c_{2 h-1}\left(\bar{\Theta}^{(2 h-1)}\right) \\
& =(2 h-1) \int_{0}^{1} c_{2 h-1}\left(\theta, \bar{\Theta}_{t}^{(2 h-2)}\right) d t
\end{aligned}
$$

where $f_{\Delta^{1}}$ means an integration over the fiber of the $\Delta^{1}$-bundle $M \times \Delta^{1} \rightarrow M$.

By Chern and Simons [CS], one obtains

$$
d\left(T(\theta) c_{2 h-1}\right)=\Delta(\theta) c_{2 h-1}=0,
$$

and the de Rham cohomology classes $\left[T(\theta) c_{2 h-1}\right] \in H_{\mathrm{DR}}^{4 h-3}\left(U_{J}(E)\right) \cong$ $H^{4 h-3}\left(U_{J}(E) ; \mathbf{R}\right)$ does not depend on the choice of an $O(n)$-connection $\theta$. This fact is due to the $\Delta^{1}$-bundle Stokes formula. These cohomology classes are called the Chern-Simons-Maslov classes of $(E, J, L)$ and are denoted by $\mu^{h}(E, J, L)$.

\section{THE $U(n)$-BUNDLE $U(n) \times_{O(n)} U(n)$ ON $M_{0}$}

The principal $O(n)$-bundle $U(n) \rightarrow U(n) / O(n)=M_{0}$ determines an $n$ dimensional Euclidean vector bundle $L_{0} \rightarrow M_{0}$. This is canonically extended to an $n$-dimensional Hermitian vector bundle $\pi_{E_{0}}: E_{0} \rightarrow M_{0}$ with a complex operator $J_{0}$.

$E_{0}$ is a symplectic $2 n$-vector bundle with the skew 2-form $\Omega_{0}(v, w)=$ - $\operatorname{Im} h_{0}(v, w)$ where $h_{0}$ is the Hermitian form of $E_{0}, \operatorname{Im} h_{0}$ is its imaginary part, and $v, w$ are vectors of $E_{0} . L_{0}$ is identified with a Lagrangian subbundle of $E_{0}$.

The principal $U(n)$-bundle associated with $E_{0}$ is

$$
\pi_{0}: U_{J_{0}}\left(E_{0}\right)=U(n) \times_{O(n)} U(n) \rightarrow M_{0} .
$$

By the section $s_{0}: M_{0} \rightarrow U(n) \times_{O(n)} U(n)$ induced from the off-diagonal map $U(n) \rightarrow U(n) \times U(n), g \mapsto\left(g, g^{-1}\right)$, it follows that the principal $U(n)$-bundle $U_{J_{0}}\left(E_{0}\right)$ is trivial; that is, we have a bundle isomorphism

$$
U(n) \times_{O(n)} U(n) \cong M_{0} \times U(n) .
$$

Under this isomorphism, the $O(n)$-subbundle associated with $L_{0}$ goes over to $\bar{\delta}(U(n)) \subset M_{0} \times U(n)$ according to the reduction argument of a structural group, where the map $\bar{\delta}: U(n) \rightarrow M_{0} \times U(n)$ is defined by $\bar{\delta} g=\left(\pi_{0}(g), g\right)$. Indeed, $\bar{\delta}$ is a bundle map covering the diagonal map $\delta$ of $M_{0}$, and we obtain a commutative diagram:

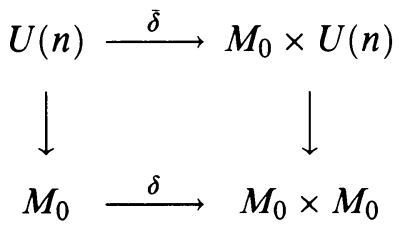

Let $\mathfrak{u}(n)$ be the Lie algebra of $U(n)$ and $\mathfrak{o}(n)$ that of $O(n)$. Let $\omega$ be the $\mathfrak{u}(n)$-valued Maurer-Cartan form on $U(n)$ and $\omega_{0}$ its $\mathfrak{o}(n)$-component. $\omega_{0}$ defines an $O(n)$-connection on the $O(n)$-bundle $U(n) \rightarrow M_{0}$. Let $\theta$ be the natural extension of $\omega_{0}$ on the $U(n)$-bundle $U_{J_{0}}\left(E_{0}\right)$. 
Lemma 3. $A \mathfrak{u}(n)$-valued 1-form $\omega-\omega_{0}$ on $U_{J_{0}}\left(E_{0}\right)$ is the $O(n)$-basic part of $\omega$.

Proof. Since $\theta$ is the extension of $\omega_{0}$, it follows that for $X \in T(U(n))$

$$
\begin{aligned}
\omega_{0}(X) & =\bar{\delta}^{*} \theta(X)=\theta\left(\bar{\delta}_{*}(X)\right)=\theta\left(\left(\pi_{0_{*}}(X), X\right)\right) \\
& =\theta\left(\left(\pi_{0_{*}}(X), 0\right)\right)+\theta((0, X))=\theta\left(s_{0_{*}} \pi_{0_{*}}(X)\right)+\omega(X) .
\end{aligned}
$$

Therefore, we get

$$
\left(\omega-\omega_{0}\right)(X)=\omega(X)-\omega_{0}(X)=-\pi_{0}^{*} s_{0}^{*} \theta(X),
$$

which means that $\omega-\omega_{0}$ is annihilated by $\mathfrak{o}(n)$ and invariant under the right translation by $O(n)$.

Since $\omega_{0}$ is obviously flat, its $U(n)$-extension $\theta$ is flat too, and hence the curvature $\boldsymbol{\theta}$ of $\theta$ is 0 . Thus it follows that

$$
\begin{aligned}
T(\theta) c_{2 h-1}= & \frac{(-1)^{h+1} \sqrt{-1}(2 h-1)}{(2 h-1) !(2 \pi)^{2 h-1}} \int_{0}^{1}\left\{\sum \delta_{i_{1} \cdots i_{2 h-1}}^{j_{1} \cdots j_{2 h-1}} \theta_{j_{1}}^{i_{1}} \wedge \overline{\boldsymbol{\Theta}}_{j_{2}}^{i_{2}} \wedge \cdots \wedge \overline{\boldsymbol{\Theta}}_{j_{2 h-1}}^{i_{2 h-1}}\right\} \wedge d t \\
= & \frac{(-1)^{h+1} \sqrt{-1}(2 h-1)}{(2 h-1) !(2 \pi)^{2 h-1}} \\
& \times \int_{0}^{1}\left\{\sum \delta_{i_{1} \cdots i_{2 h-1}}^{j_{1} \cdots j_{2 h-1}} \theta_{j_{1}}^{i_{1}} \wedge\left(t \Theta_{j_{2}}^{i_{2}}+t(t-1)[\theta, \theta]_{j_{2}}^{i_{2}}\right)\right. \\
& \left.\wedge \cdots \wedge\left(t \Theta_{j_{2 h-1}}^{i_{2 h-1}}+t(t-1)[\theta, \theta]_{j_{2 h-1}}^{i_{2 h-1}}\right)\right\} \wedge d t \\
= & \frac{(2 h-1) !(2 h-1) !}{(4 h-3) !} \frac{(-1)^{h+1} \sqrt{-1}}{(2 h-1) !(2 \pi)^{2 h-1}} \\
& \times\left\{\sum \delta_{i_{1} \cdots i_{2 h-1}}^{j_{1} \cdots j_{2 h-1}} \theta_{j_{1}}^{i_{1}} \wedge[\theta, \theta]_{j_{2}}^{i_{2}} \wedge \cdots \wedge[\theta, \theta]_{j_{2 h-1}}^{i_{2 h-1}}\right\} \\
= & \Delta(\theta) \sigma c_{2 h-1},
\end{aligned}
$$

where $\sigma c_{k}$ is of the form

$$
\frac{(k-1) ! k !}{(2 k-1) !} c_{k}(l \wedge \underbrace{[l, l] \wedge \cdots \wedge[l, l]}_{k-1})
$$

for the identity 1 -form $l$ on $u(n)$ and is denoted by $y_{k}$.

It is well known that $H^{*}(U(n) ; \mathbf{R}) \cong H^{*}(\mathfrak{u}(n)) \cong \Lambda\left(y_{1}, \ldots, y_{n}\right)$ and for $n^{\prime}=2[(n+1) / 2]-1$ that

$$
H^{*}(U(n) / O(n) ; \mathbf{R}) \cong H^{*}(\mathfrak{u}(n), O(n)) \cong \bigwedge\left(y_{1}, y_{3}, \ldots, y_{n^{\prime}}\right) .
$$

Hereinafter, we denote $y_{2 h-1}$ in $H^{*}(U(n) / O(n) ; \mathbf{R})$ by $\Delta_{*} y_{2 h-1}$.

3. $y_{k}, \Delta_{*} y_{2 h-1}$, AND THE CLASS $\mu^{1}\left(E_{0}, J_{0}, L_{0}\right)$

In the trivialization, $U_{J_{0}}\left(E_{0}\right) \cong M_{0} \times U(n)$, let $i_{0}: U(n) \rightarrow U_{J_{0}}\left(E_{0}\right)$ be the inclusion map defined by $g \mapsto\left(\left[I_{n}\right], g\right)$ for $g \in U(n)$, where $I_{n}$ is the unit element of $U(n)$. 
Lemma 4. Let $\theta$ be the natural extension of the $O(n)$-connection $\omega_{0}$ on $U(n) \rightarrow$ $M_{0}$ to $U_{J_{0}}\left(E_{0}\right)$. We have $s_{0}^{*}\left[T(\theta) c_{2 h-1}\right]=-\Delta_{*} y_{2 h-1}$ and $i_{0}^{*}\left[T(\theta) c_{k}\right]=y_{k}$.

Proof. Since $\pi_{0}^{*} s_{0}^{*} \theta$ is the $O(n)$-basic part of $\omega$ by Lemma 3, it follows that

$$
s_{0}^{*}\left[T(\theta) c_{2 h-1}\right]=\left[T\left(s_{0}^{*} \theta\right) c_{2 h-1}\right]=s_{0}^{*}\left[T\left(\pi_{0}^{*} s_{0}^{*} \theta\right) c_{2 h-1}\right]=-\Delta_{*} y_{2 h-1} \text {. }
$$

Since $\theta$ is the $U(n)$-connection, we have

$$
i_{0}^{*}\left[T(\theta) c_{k}\right]=\left[T\left(i_{0}^{*} \theta\right) c_{k}\right]=\left[T(\omega) c_{k}\right]=y_{k} .
$$

It is known that $\pi_{1}(U(n)) \cong \mathbf{Z}$ and $\pi_{1}(U(n) / O(n)) \cong \mathbf{Z}$ (see, e.g., [M]), and hence we get $H^{1}(U(n)) \cong \mathbf{Z}$ and $H^{1}(U(n) / O(n)) \cong \mathbf{Z}$.

Proof of Theorem 1. By the Künneth formula for cohomology rings (see, e.g., [S] $)$, we have $H^{1}\left(U_{J_{0}}\left(E_{0}\right) ; \mathbf{R}\right) \cong H^{1}\left(M_{0} \times U(n) ; \mathbf{R}\right) \cong H^{1}\left(M_{0} ; \mathbf{R}\right) \otimes 1+1 \otimes$ $H^{1}(U(n) ; \mathbf{R})$, and the identification formula for $\mu \in H^{1}\left(U_{J_{0}}\left(E_{0}\right) ; \mathbf{R}\right)$ under this isomorphism is $\mu=s_{0}^{*} \mu \otimes 1+1 \otimes i_{0}^{*} \mu$. It follows that

$$
s_{0}^{*} \mu^{1}\left(E_{0}, J_{0}, L_{0}\right)=s_{0}^{*}\left[T(\theta) c_{1}\right]=\left[\frac{\sqrt{-1}}{2 \pi} \operatorname{trace}\left(s_{0}^{*} \theta\right)\right] \text {. }
$$

The closed path $\gamma:[0, \pi] \rightarrow U(n) / O(n)$ defined by $\gamma(t)=\left[e^{\sqrt{-1} t} \oplus I_{n-1}\right]$ represents the canonical generator $a \in H_{1}(U(n) / O(n))$. Let $\tilde{\gamma}$ be a lift of $\gamma$ to $U(n)$. Then we have

$$
\begin{aligned}
{\left[\frac{\sqrt{-1}}{2 \pi} \operatorname{trace}\left(s_{0}^{*} \theta\right)\right](a) } & =\frac{\sqrt{-1}}{2 \pi} \int_{\gamma} \operatorname{trace}\left(s_{0}^{*} \theta\right)=-\frac{\sqrt{-1}}{2 \pi} \int_{\tilde{\gamma}} \operatorname{trace} \omega \\
& =-\frac{\sqrt{-1}}{2 \pi} \int_{0}^{\pi} \sqrt{-1} d t=\frac{1}{2},
\end{aligned}
$$

and hence $s_{0}^{*} \mu^{1}\left(E_{0}, J_{0}, L_{0}\right)=-\frac{1}{2} u$.

Similarly, it follows that

$$
i_{0}^{*} \mu^{1}\left(E_{0}, J_{0}, L_{0}\right)=i_{0}^{*}\left[T(\theta) c_{1}\right]=\left[T(\omega) c_{1}\right]=-v .
$$

Thus one obtains

$$
\begin{aligned}
\mu^{1}\left(E_{0}, J_{0}, L_{0}\right) & =s_{0}^{*} \mu^{1}\left(E_{0}, J_{0}, L_{0}\right) \otimes 1+1 \otimes i_{0}^{*} \mu^{1}\left(E_{0}, J_{0}, L_{0}\right) \\
& =\frac{1}{2}(u \otimes 1-2 \otimes v) .
\end{aligned}
$$

\section{The Classes $\mu^{h}(E, J, L)$}

Let $\pi: E \rightarrow M$ be a symplectic $2 n$-vector bundle, $J$ a compatible complex structure, and $L \subset E$ a Lagrangian subbundle. Suppose that $E$ is trivial as the complex $n$-vector bundle with respect to $J$. Then it is classified by a map $f: M \rightarrow U(n) / O(n)$, and we have the commutative diagram

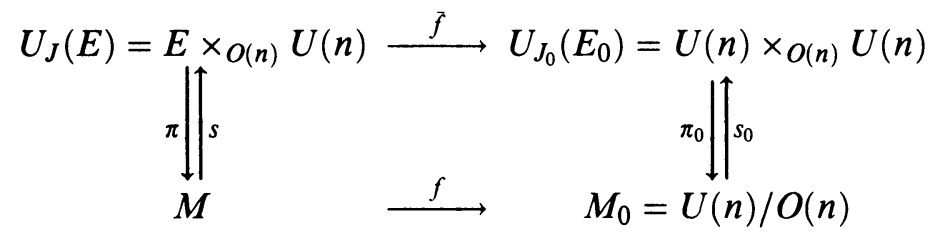

where $\bar{f}$ is the bundle space map covering $f$ and $s$ is the section pulled back from $s_{0}$ by $f$.

High-dimensional Maslov classes for $(E, J, L)$ in the sense of Kamber and Tondeur [KT] are the pullbacks $\Delta_{E *} y_{2 h-1}=f^{*} \Delta_{*} y_{2 h-1} \in H^{4 h-3}(M ; \mathbf{R})$. 
Proposition 5. We have $s^{*} \mu^{h}(E, J, L)=-\Delta_{E *} y_{2 h-1}$.

Proof. The pullback $\bar{f}^{*} \theta$ is a flat connection on $U_{J}(E)$. From the commutativity of the above diagram and the first formula of Lemma 4 , it follows that

$$
\begin{aligned}
s^{*} \mu^{h}(E, J, L) & =s^{*}\left[T\left(\bar{f}^{*} \theta\right) c_{2 h-1}\right]=s^{*} \bar{f}^{*}\left[T(\theta) c_{2 h-1}\right]=f^{*} s_{0}^{*}\left[T(\theta) c_{2 h-1}\right] \\
& =-f^{*} \Delta_{*} y_{2 h-1}=-\Delta_{E *} y_{2 h-1} .
\end{aligned}
$$

Proposition 5 shows that the Maslov classes $\Delta_{E *} y_{2 h-1}$ of Kamber and Tondeur are pullbacks of $-\mu^{h}(E, J, L)$ by the section of trivialization.

Proof of Theorem 2. We take a classifying map $f: M \rightarrow U(n) / O(n)$ for the $U(n)$-bundle $\pi: U_{J}(E) \rightarrow M$ such that $f\left(x_{b}\right)=\left[I_{n}\right]$ for a fixed point $x_{b} \in M$. Let $i: U(n) \rightarrow U_{J}(E)$ be the inclusion map to the fiber over $x_{b}$. The section $s$ gives a trivialization $U_{J}(E) \cong M \times U(n)$. One gets a commutative diagram:

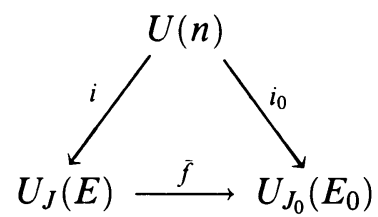

It follows that

$$
\begin{aligned}
i^{*} \mu^{h}(E, J, L) & =i^{*}\left[T\left(\bar{f}^{*} \theta\right) c_{2 h-1}\right]=\left[T\left(i^{*} \bar{f}^{*} \theta\right) c_{2 h-1}\right]=\left[T\left(i_{0}^{*} \theta\right) c_{2 h-1}\right] \\
& =\left[T(\omega) c_{2 h-1}\right]=y_{2 h-1},
\end{aligned}
$$

as the second formula of Lemma 4.

In the cohomology ring $H^{*}\left(U_{J}(E) ; \mathbf{R}\right) \cong H^{*}(M ; \mathbf{R}) \otimes H^{*}(U(n) ; \mathbf{R})$, the $(4 h-3,0)$-component of $\mu^{h}(E, J, L)$ is $s^{*} \mu^{h}(E, J, L) \otimes 1=-\Delta_{E *} y_{2 h-1} \otimes$ 1 by Proposition 5 and the $(0,4 h-3)$-component of $\mu^{h}(E, J, L)$ is $1 \otimes$ $i^{*} \mu^{h}(E, J, L)=1 \otimes y_{2 h-1}$. The middle components of $\mu^{h}(E, J, L)$ all vanish by our assumption. Thus one gets

$$
\mu^{h}(E, J, L)=-\Delta_{E *} y_{2 h-1} \otimes 1+1 \otimes y_{2 h-1} .
$$

\section{REFERENCES}

[CS] S. S. Chern and J. Simons, Characteristic forms and geometric invariants, Ann. of Math. (2) 99 (1974), 48-69.

[KT] F. Kamber and Ph. Tondeur, Foliated bundles and characteristic classes, Lecture Notes in Math., vol. 493, Springer, Berlin and New York, 1975.

[M] J. Milnor, Morse theory, Princeton Univ. Press, Princeton, NJ, 1963.

[S] E. H. Spanier, Algebraic topology, McGraw-Hill, New York, 1966.

[V] I. Vaisman, Symplectic geometry and secondary characteristic classes, Birkhäuser, Boston and Basel, 1987.

Department of Mathematics, Hokkaido University, Sapporo 060, Japan

E-mail address: C12161@sinet.ad.jp

E-mail address: suzuki@euler.math.hokudai.ac.jp 